\title{
Transfer matrix method applied to the parallel assembly of sound absorbing materials
}

\author{
Kévin Verdière, ${ }^{a)}$ Raymond Panneton, and Saïd Elkoun \\ GAUS, Department of Mechanical Engineering, Université de Sherbrooke (Qc), JIK 2RI, Canada \\ Thomas Dupont and Philippe Leclaire \\ DRIVE, ISAT, Université de Bourgogne, 49 Rue Mademoiselle Bourgeois, 58027 Nevers Cedex, France
}

\begin{abstract}
The transfer matrix method (TMM) is used conventionally to predict the acoustic properties of laterally infinite homogeneous layers assembled in series to form a multilayer. In this work, a parallel assembly process of transfer matrices is used to model heterogeneous materials such as patchworks, acoustic mosaics, or a collection of acoustic elements in parallel. In this method, it is assumed that each parallel element can be modeled by a $2 \times 2$ transfer matrix, and no diffusion exists between elements. The resulting transfer matrix of the parallel assembly is also a $2 \times 2$ matrix that can be assembled in series with the classical TMM. The method is validated by comparison with finite element (FE) simulations and acoustical tube measurements on different parallel/series configurations at normal and oblique incidence. The comparisons are in terms of sound absorption coefficient and transmission loss on experimental and simulated data and published data, notably published data on a parallel array of resonators. From these comparisons, the limitations of the method are discussed. Finally, applications to threedimensional geometries are studied, where the geometries are discretized as in a FE concept. Compared to FE simulations, the extended TMM yields similar results with a trivial computation time.
\end{abstract}

\section{INTRODUCTION}

In our modern society, noise pollution is a serious concern as it has a profound impact on the economy, health, and productivity. Thus, the need to reduce noise has led to the development of a wide range of sound barriers and/or absorbing materials. Among the most used sound-absorbing materials, porous materials exhibit, in general, fairly high sound absorption coefficients. However, their acoustic performances are highly dependent upon frequency and are still poor at low frequency. To overcome this problem, different porous materials can be stacked to obtain porous multilayered materials. Knowing the properties of each layer, the acoustical behavior of multilayered materials can easily be predicted using the transfer matrix method (TMM) for materials stacked in series. ${ }^{1}$ In contrast, for parallel stacking of materials, the Finite Element Method (FEM) is the most general approach to derive acoustic properties. For instance, the acoustic properties of micro-perforated panels (MPPs) with different parallel cavity sizes, ${ }^{2}$ parallel tubes in mufflers, ${ }^{3}$ parallel periodic structures, ${ }^{4,5}$ and parallel assembly of porous material ${ }^{6}$ can be obtained using FEM simulations or analytical models developed for specific cases and, therefore, limited to few applications and difficult to generalize. As far as we know, no study has been published yet using TMM for deriving the acoustic properties of multilayered materials made of parallel stacking of various materials and structures.

\footnotetext{
a) Author to whom correspondence should be addressed. Electronic mail: Kevin.Verdiere@USherbrooke.ca
}

In electrical engineering, transfer matrices are widely used to model portions of circuits that are connected together. Transfer matrices are not only used to describe series and parallel circuits but combinations of these matrices are frequently used to solve various problems. ${ }^{7}$

The aim of this paper is to give an extension of the TMM to account for the assembly of materials (or acoustic elements) in parallel and series and not in series only. In this work, the parallel arrangement is also referred to as patchwork or acoustic mosaic and its development is based on a method reported in the literature to simulate dead-end pores in aluminum foams. ${ }^{8}$ The proposed method is generalized and validated through examples and its limits are discussed and applied to complex three-dimensional (3D) geometries.

\section{THEORY}

The TMM is regarded as a powerful method to predict sound absorption and sound transmission of multilayered materials. ${ }^{1}$ It is mainly used to obtain the sound absorption coefficient and transmission loss of assemblies consisting of laterally infinite and homogeneous material layers stacked in series.

In this work, a method for assembling transfer matrices of elements stacked in parallel is developed. This parallel arrangement of elements is also referred to as a patchwork construction or an acoustic mosaic. An example is shown in Fig. 1. The construction is defined by a periodic elementary patchwork shown in bold in Fig. 1. The key assumptions underlying the method are: (1) Only plane waves propagate upstream and downstream of the construction; (2) only normal incidence plane waves propagate in the construction; (3) no 


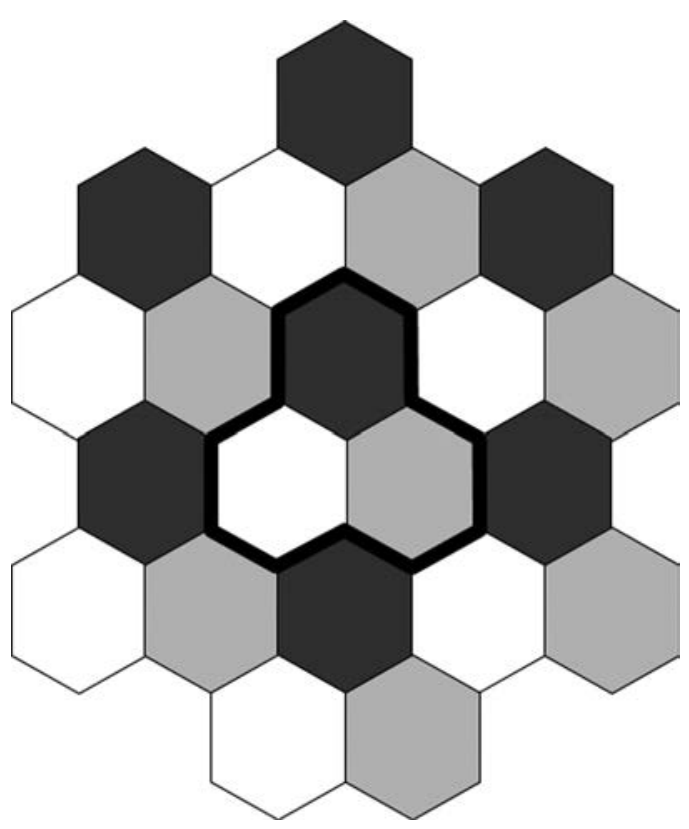

FIG. 1. Example of a patchwork construction, or acoustic mosaic, made from a periodic assembly of parallel acoustical elements (e.g., Helmholtz or quarter wavelength resonator) or materials (e.g., porous or fibrous material). The periodic elementary patchwork is shown in bold. In this example, the elementary patchwork is composed of $N=3$ elements.

pressure diffusion exists between adjacent parallel elements, (4) the wavelength is much larger than the periodic elementary patchwork, and (5) each element can be represented by a $2 \times 2$ transfer matrix. Following the latter assumption, elements could be either perforated plates, fluids, porous materials modeled as equivalent fluids, ${ }^{9}$ a serial assembly of the aforementioned elements, a measured $2 \times 2$ transfer matrix, ${ }^{10}$ or whatever that can be expressed by a $2 \times 2$ transfer matrix.

The two-dimensional (2D) schematic representation of the studied parallel construction is depicted in Fig. 2. It consists of the stacking of $N$ elements arranged in parallel separating two air domains. Following assumption (2), each element may be seen as being locally reacting and a waveguide along the $x$ axis. Each element can consist of a single material or a stack of materials in series. To link the acoustic pressures $P$ and the $x$-component $U$ of the acoustic velocities in the air on either side of element $i$, the following $2 \times 2$ transfer matrix relation is used:

$$
\left\{\begin{array}{l}
P\left(M_{i}\right) \\
U\left(M_{i}\right)
\end{array}\right\}=\mathbf{T}_{i}\left\{\begin{array}{l}
P\left(M_{i}^{\prime}\right) \\
U\left(M_{i}^{\prime}\right)
\end{array}\right\}=\left[\begin{array}{ll}
t_{i, 11} & t_{i, 12} \\
t_{i, 21} & t_{i, 22}
\end{array}\right]\left\{\begin{array}{l}
P\left(M_{i}^{\prime}\right) \\
U\left(M_{i}^{\prime}\right)
\end{array}\right\},
$$

where $t_{i, m n}$ are the coefficients of transfer matrix $\mathbf{T}_{i}$. Assuming element $i$ is a rigid frame open cell porous element of thickness $h$, the transfer matrix would be given by ${ }^{1}$

$$
\mathbf{T}_{i}=\left[\begin{array}{cc}
\cos \left(k_{e q, i} h\right) & j Z_{c e q, i} \sin \left(k_{e q, i} h\right) \\
j \frac{1}{Z_{c e q, i}} \sin \left(k_{e q, i} h\right) & \cos \left(k_{e q, i} h\right)
\end{array}\right]
$$

where $j^{2}=-1$, and $Z_{c e q, i}$ and $k_{e q, i}$ are the characteristic impedance and wave number of the equivalent fluid element- to be distinguished from the characteristic impedance $Z_{c, i}$ of the fluid

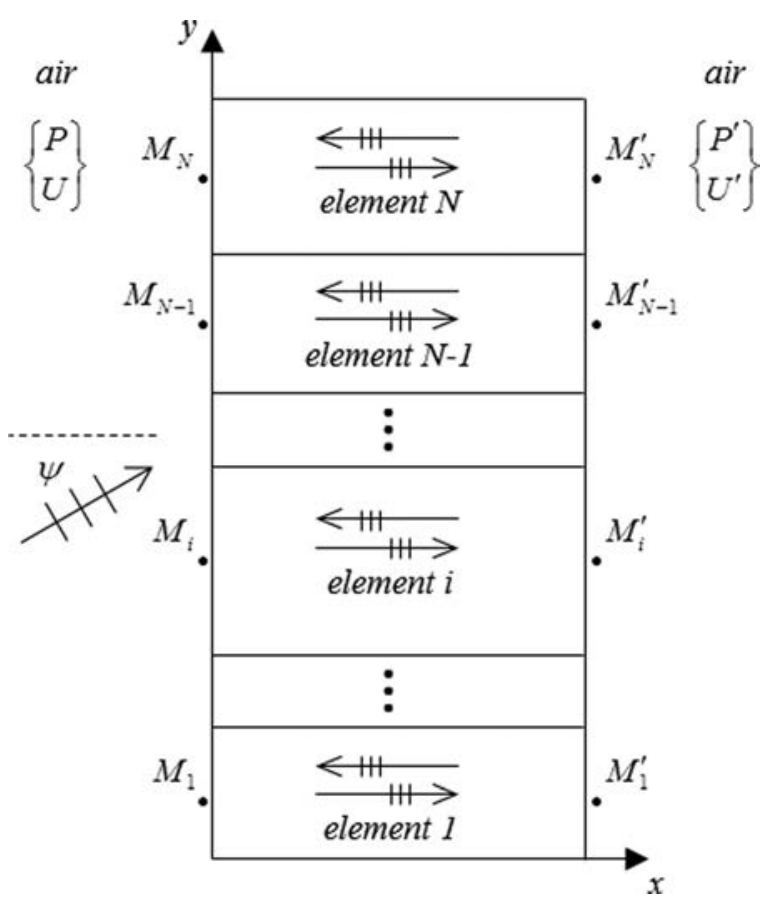

FIG. 2. Plane wave impinging on the studied parallel construction made of $N$ parallel elements. The arrows represent plane waves.

phase. ${ }^{9}$ The relation between both impedances is $Z_{c e q, i}=Z_{c, i} / \phi_{i}$, where $\phi_{i}$ is the open porosity of the element. It is worth mentioning that Eq. (2) assumes propagation parallel to the element axis to conform to assumption (2).

Since the elements are in parallel, it is more convenient to work with admittances. Consequently, Eq. (1) can be written in terms of an admittance matrix $\mathbf{Y}_{i}$ as follows:

$$
\left\{\begin{array}{l}
U\left(M_{i}\right) \\
U\left(M_{i}^{\prime}\right)
\end{array}\right\}=\mathbf{Y}_{i}\left\{\begin{array}{l}
P\left(M_{i}\right) \\
P\left(M_{i}^{\prime}\right)
\end{array}\right\}
$$

with

$$
\mathbf{Y}_{i}=\left[\begin{array}{ll}
y_{i, 11} & y_{i, 12} \\
y_{i, 21} & y_{i, 22}
\end{array}\right]=\frac{1}{t_{i, 12}}\left[\begin{array}{cc}
t_{i, 22} & t_{i, 21} t_{i, 12}-t_{i, 22} t_{i, 11} \\
1 & -t_{i, 11}
\end{array}\right] .
$$

If the reciprocity principle ${ }^{11}$ applies, the determinant of $\mathbf{T}_{i}$ is equal to unity (i.e., $t_{i, 11} t_{i, 22}-t_{i, 12} t_{i, 21}=1$ ), and $y_{i, 12}$ $=-y_{i, 21}$. In this case, up to sign, Eq. (4) shows that the admittance matrix is symmetric. This is expected since the $x$-component of the acoustic velocity was used instead of the normal acoustic velocity with normal vector inward to the construction domain as usually done following an admittance approach. Here, the $x$-component is preferred with a view to comply with the classical TMM description. ${ }^{1}$

\section{A. Case with all open elements upstream and downstream}

Consider that initially all the elements of the construction are open to air on the upstream and downstream sides. Following assumptions (1) and (4), since only plane waves propagate in the surrounding air, the pressure is uniform over the upstream and downstream faces in the air. This imposes continuity of pressure at each air-element interface. Similarly, 
continuity of flow rate is preserved at the interfaces. These continuity conditions can be written as

$$
\left\{\begin{array}{l}
P\left(M_{i}\right) \\
P\left(M_{i}^{\prime}\right)
\end{array}\right\}=\left\{\begin{array}{l}
P \\
P^{\prime}
\end{array}\right\}
$$

and

$$
\left\{\begin{array}{l}
U \\
U^{\prime}
\end{array}\right\}=\sum r_{i}\left\{\begin{array}{l}
U\left(M_{i}\right) \\
U\left(M_{i}^{\prime}\right)
\end{array}\right\}
$$

where $P$ and $U$ are the uniform acoustic fields in the incident air medium on the upstream surface of the construction, and $P^{\prime}$ and $U^{\prime}$ are the uniform acoustic fields in the transmitted air medium on its downstream surface. In Eq. (6), $r_{i}$ is the surface ratio of element $i$ given by

$$
r_{i}=S_{i} / S_{\text {total }}
$$

where $S_{i}$ and $S_{\text {total }}$ are the cross-sectional surface areas of element $i$ and the construction, respectively. Using the continuity conditions, Eqs. (5) and (6), and the admittance matrix system, Eq. (3), it follows

$$
\left\{\begin{array}{l}
U \\
U^{\prime}
\end{array}\right\}=\left(\sum r_{i}\left[\begin{array}{ll}
y_{i, 11} & y_{i, 12} \\
y_{i, 21} & y_{i, 22}
\end{array}\right]\right)\left\{\begin{array}{l}
P \\
P^{\prime}
\end{array}\right\} .
$$

This is the admittance system of the whole parallel construction. This admittance system can be reformulated in terms of a transfer matrix system as

$$
\left\{\begin{array}{l}
P \\
U
\end{array}\right\}=\mathbf{T}_{\mathbf{p}}\left\{\begin{array}{l}
P^{\prime} \\
U^{\prime}
\end{array}\right\}
$$

with

$$
\begin{aligned}
& \mathbf{T}_{\mathbf{p}}=\frac{-1}{\sum r_{i} y_{i, 21}} \\
& \left.\times \quad \begin{array}{cc}
\Sigma r_{i} y_{i, 22} & -1 \\
\Sigma r_{i} y_{i, 22} \Sigma r_{i} y_{i, 11}-\Sigma r_{i} y_{i, 12} \Sigma r_{i} y_{i, 21} & -\Sigma r_{i} y_{i, 11}
\end{array}\right),
\end{aligned}
$$

where $\mathbf{T}_{\mathbf{p}}$ is the transfer matrix of the parallel construction when all the elements are open at their ends. This transfer matrix relates the acoustic fields in the air before the construction to the acoustic fields in the air behind the material.

\section{B. Case with closed elements downstream}

Consider the particular case where some elements are closed on the downstream side-the rest being open to air. In this case, each element must be clearly identifiable. Accordingly, subscript $j$ will stand for open-ended elements while subscript $k$ will refer to close-ended elements. While the continuity conditions on the flow rate and the upstream pressure remain unchanged, the continuity of pressure on the downstream side is only ensured for the open-ended elements. Consequently, Eq. (5) becomes

$$
\left\{\begin{array}{l}
P\left(M_{i}\right) \\
P\left(M_{j}^{\prime}\right)
\end{array}\right\}=\left\{\begin{array}{l}
P \\
P^{\prime}
\end{array}\right\} .
$$

For element $k$ closed to end, the normal velocity vanishes at $M_{k}^{\prime}$, and the admittance system, Eq. (3), yields the following condition:

$$
P\left(M_{k}^{\prime}\right)=-\frac{y_{k, 21}}{y_{k, 22}} P
$$

Finally, replacing Eq. (5) by Eqs. (11) and (12), and applying the same procedure, the transfer matrix of the parallel construction can be rewritten as

$$
\mathbf{T}_{\mathbf{p}}=\frac{-1}{\Sigma r_{j} y_{j, 21}}\left(\begin{array}{cc}
\Sigma r_{j} y_{j, 22} & -1 \\
\Sigma r_{j} y_{j, 22}\left(\Sigma r_{i} y_{i, 11}-\Sigma r_{k} \frac{y_{k, 12} y_{k, 21}}{y_{k, 22}}\right)-\Sigma r_{j} y_{j, 12} \Sigma r_{j} y_{j, 21} & \Sigma r_{k} \frac{y_{k, 12} y_{k, 21}}{y_{k, 22}}-\Sigma r_{i} y_{i, 11}
\end{array}\right) .
$$

This time, $\mathbf{T}_{\mathbf{p}}$ is the transfer matrix of the parallel construction where some elements are open to air on the downstream side, and some are closed. One can verify that if there is no close-ended elements (i.e., subscript $k$ vanishes and subscript $j$ is identical to $i$ ), then Eq. (13) is identical to Eq. (10). A similar development can be done for the case where some elements are closed on the upstream side, or on both sides.

While Eq. (13) seems to be general, one needs to pay attention to the case where all elements are close-ended (i.e., no $j$ subscript). In this case, Eq. (13) does not hold due a division by 0 in the term before the matrix. As a matter of fact, to use Eq. (13), at least one element must be openended. In the situation where all elements are closed to end (i.e., the construction is backed by a rigid wall), one should use Eq. (10) to retrieve the acoustic properties. This is similar to the classical TMM and described in Sec. II C.

Also, it is worth noting that if all elements of the construction are reciprocal, then both systems [Eqs. (10) and (13)] are in line with the reciprocity principle which stipulates 
that $\operatorname{det}\left(\mathbf{T}_{\mathbf{p}}\right)=1$. However, if all elements respect the material symmetry condition $t_{11}=t_{22}$ (i.e., same acoustical surface properties on both sides), then only the transfer matrix of Eq. (10) shows the same symmetry condition.

Finally, it is highlighted that each porous element is counter-balanced by its surface ratio, and matrix $\mathbf{T}_{\mathbf{p}}$ is the same whatever the element position. Moreover, the construction of matrix $\mathbf{T}_{\mathbf{p}}$ does not require to take into account or to know the number of similar entities that composed the patchwork construction. Indeed and for instance, a patchwork made of four equal elements, where two elements are filled with material $\mathrm{A}$ and the other two with material $\mathrm{B}$, can be regarded as composed of two elements of material $\mathrm{A}$ and two elements of material B or, because of assumption (4), one element of material A $\left(r_{1}=50 \%\right)$ and one element of material B $\left(r_{2}=50 \%\right)$. In fact, with assumption (4), the acoustic wavelength is supposed to be much larger than the periodic elementary patch (or mosaic characteristic size) and thus can be considered as homogeneous. The validation of this approach is discussed in the experimental part of the present study.

\section{Coupling parallel and serial constructions}

The developed transfer matrix for parallel constructions can be assembled in series with other homogeneous layers following the classical method. As an example, consider an air cavity of thickness $L$ modeled by the following $2 \times 2$ transfer:

$$
\mathbf{T}_{\text {air }}=\left[\begin{array}{cc}
\cos \left(k_{0} L \cos \psi\right) & j \frac{Z_{0}}{\cos \psi} \sin \left(k_{0} L \cos \psi\right) \\
j \frac{\cos \psi}{Z_{0}} \sin \left(k_{x} L\right) & \cos \left(k_{0} L \cos \psi\right)
\end{array}\right],
$$

where $Z_{0}$ and $k_{0}$ are the characteristic impedance and wave number in air, and $\psi$ is the angle of incidence of the plane wave in air. If the air cavity is backing the patchwork, the global transfer matrix of the parallel/serial construction is

$$
\left\{\begin{array}{l}
P \\
U
\end{array}\right\}=\mathbf{T}_{\mathbf{G}}\left\{\begin{array}{l}
P^{\prime} \\
U^{\prime}
\end{array}\right\} \text { with } \mathbf{T}_{\mathbf{G}}=\mathbf{T}_{\mathbf{p}} \mathbf{T}_{\mathbf{a i r}}=\left[\begin{array}{ll}
T_{11} & T_{12} \\
T_{21} & T_{22}
\end{array}\right] .
$$

From this global transfer matrix, one can calculate the hardbacking normal incidence sound absorption coefficient and the anechoic-end normal incidence sound transmission loss by, respectively,

$$
\alpha=1-\left|\frac{T_{11} \cos \Psi-T_{21} Z_{0}}{T_{11} \cos \Psi-T_{21} Z_{0}}\right|^{2}
$$

and

$$
\left.N S T L=20 \log _{10} \frac{1}{2}\left|T_{11}+T_{22}+\frac{\cos \psi}{Z_{0}} T_{12}+\frac{Z_{0}}{\cos \psi} T_{21}\right|\right) .
$$

TABLE I. Physical properties of selected materials.

\begin{tabular}{lccccccc}
\hline \hline & Material & $\phi$ & $\sigma\left(\mathrm{N} \mathrm{s} \mathrm{m}^{-4}\right)$ & $\Lambda(\mu \mathrm{m})$ & $\Lambda \prime(\mu \mathrm{m})$ & $\alpha_{\infty}$ & $\rho_{1}\left(\mathrm{~kg} / \mathrm{m}^{3}\right)$ \\
\hline A & Melamine foam & 0.999 & 9724 & 110 & 122 & 1.00 & 8.3 \\
$\mathrm{~B}$ & Glass wool & 0.999 & 15957 & 97 & 530 & 1.00 & 40 \\
$\mathrm{C}$ & Rock wool & 0.960 & 41554 & 92 & 92 & 2.59 & 90 \\
$\mathrm{D}$ & Rock wool & 0.940 & 135000 & 49 & 166 & 2.10 & - \\
\multicolumn{2}{c}{ (diffusion) (Ref. 13) } & & & & & & \\
$\mathrm{E}$ & Polyurethane foam & 0.958 & 11188 & 70 & 209 & 1.94 & 29 \\
\hline \hline
\end{tabular}

Note that if the absorption and transmission loss of a patchwork construction is studied only, the transfer matrix coefficients to use in Eqs. (16) and (17) are those given by $\mathbf{T}_{\mathbf{p}}$.

\section{MATERIALS AND METHODS}

To obtain the transfer matrix of the patchwork construction, the transfer matrix of each element must be known. For the validation results presented in Sec. IV, only rigid or limp frame open-cell porous elements are considered. Consequently, transfer matrices of Eq. (2) are used. The equivalent fluid properties $Z_{c e q}$ and $k_{e q}$ populating Eq. (2) are computed by the use of the Johnson-Champoux-Allard model. ${ }^{1}$ This model requires five parameters: Porosity, static airflow resistivity, tortuosity, viscous, and thermal characteristic lengths (or six parameters for limp frame ${ }^{9}$ with the additional parameter being the bulk density of the porous aggregate). These properties are obtained as described by Doutres et al. ${ }^{12}$ Table I depicts the selected porous materials and their main physical characteristics. Except for material D, all properties have been experimentally assessed following Ref. 12. For material D, where diffusion phenomena have been evidenced, data published by Olny and Boutin have been used. ${ }^{13}$

The predictions obtained with the TMM are systematically compared to impedance/transmission tube measurements. The schematic of the tube is shown in Fig. 3. It consists of a $44.5 \mathrm{~mm}$ or a $100 \mathrm{~mm}$ diameter tube supporting three microphones. The cut-off (maximum) frequencies of the tubes are 4100 and $1800 \mathrm{~Hz}$, respectively. From pressure measurements at the three microphones, normal incidence (i.e., $\psi=0$ ) sound absorption coefficient and sound transmission loss are deduced according to the three microphone method $^{10}$ and standard ASTM E2611-09. ${ }^{14}$ All experimental data are compared to virtual measurements obtained with $3 \mathrm{D}$ acoustical FEM simulations using COMSOL software. The FEM model is a reproduction of the setup shown in Fig. 3. In

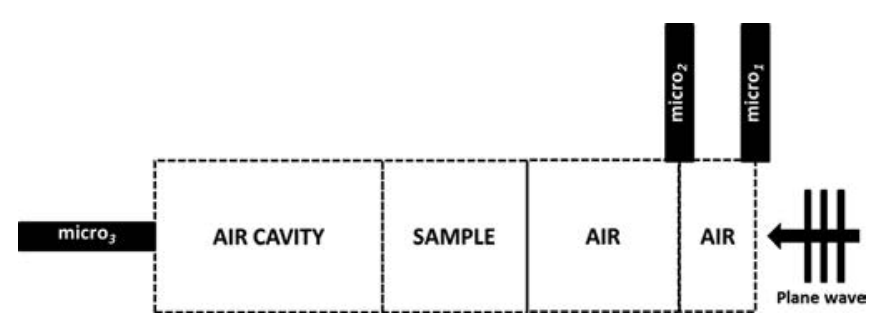

FIG. 3. Experimental and FEM model diagram of the three-microphone impedance/transmission tube. 

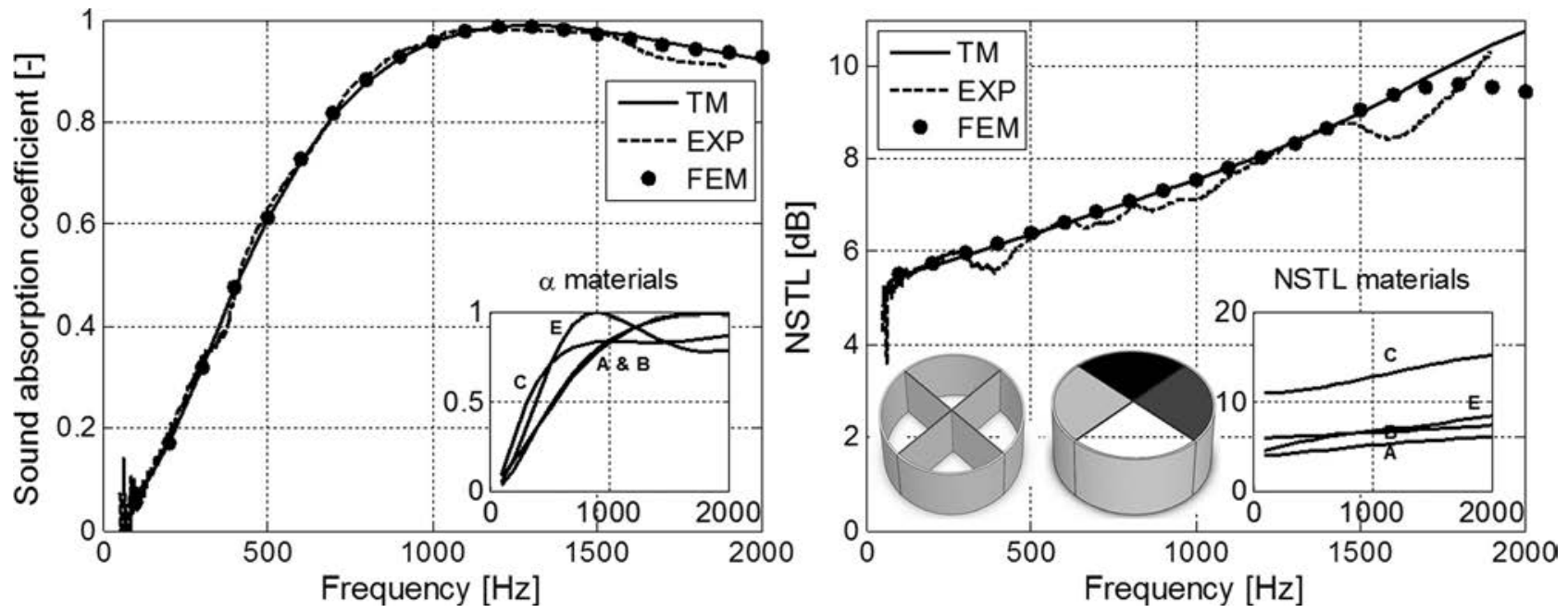

FIG. 4. Normal incidence sound absorption coefficient and transmission loss of a parallel assembly of four materials: Melamine foam (A), glass wool (B), rock wool (D), and polyurethane foam (E). The four materials are inserted in an impervious cross grid. Comparison between TMM, FEM, and experimental results. Acoustic parameters of each material composing the assembly are inset.

the FEM model on COMSOL, parabolic tetrahedral elements are used to mesh the different acoustic domains of the tube, and convergence of the results is verified in the frequency range of interest. Also, the porous elements of the construction samples are modeled as equivalent fluids using complex sound speed ( $c_{e q}=\omega / k_{e q}$, where $\omega$ is the angular frequency) and dynamic density $\left(\rho_{e q}=Z_{c e q} k_{e q} / \omega\right)$ given by the Johnson-Champoux-Allard model previously described.

\section{RESULTS AND DISCUSSION}

\section{A. Parallel construction}

\section{Normal incidence}

The first construction studied is a cylindrical assembly of $N=4$ materials in parallel under a normal incidence plane wave $(\psi=0)$. The materials are the melamine foam, glass wool, rock wool, and polyurethane foam corresponding to materials A, B, C, and E of Table I. Each material represents $25 \%$ of the total surface and the cylinder is longitudinally split into four pieces. The cylinder is $50 \mathrm{~mm}$ thick and its diameter is $100 \mathrm{~mm}$. For the impedance tube test, with a view to respect assumption (3) of the TMM (no pressure diffusion between elements), each material is isolated by an ABS plastic cross grid inserted in the tube, see Fig. 4. The same setup is used for the FEM simulation. Figure 4 depicts the absorption coefficient and the transmission loss of this parallel construction as a function of frequency. One can notice that both acoustic properties obtained by the TMM are consistent with data predicted by the FEM simulation and experiment. However a divergence between the TMM and FEM data can be observed at frequencies above $1600 \mathrm{~Hz}$. This is due to the fact that the grid creates a sound field disturbance that lowers the cutoff frequency of the tube from 1800 to $1600 \mathrm{~Hz}$. A modal analysis with the FEM model has confirmed this. To improve the validity of the TMM, insets of acoustic properties of each material which compose the parallel assembly are added in Fig. 4. As you can see, these properties are clearly different from those of the construction.

\section{Oblique incidence}

The acoustic properties of the previous construction are now obtained under oblique incidence. Since no experimental setup is available for the oblique incidence test, the TMM is compared to FEM simulation only. The FEM model for oblique incidence is shown in Fig. 5. A sample is excited by an oblique plane wave. Since the sample reacts locally [assumption (2)], the wave is guided along the normal to its surface, comes out at the same incidence angle, and reflects

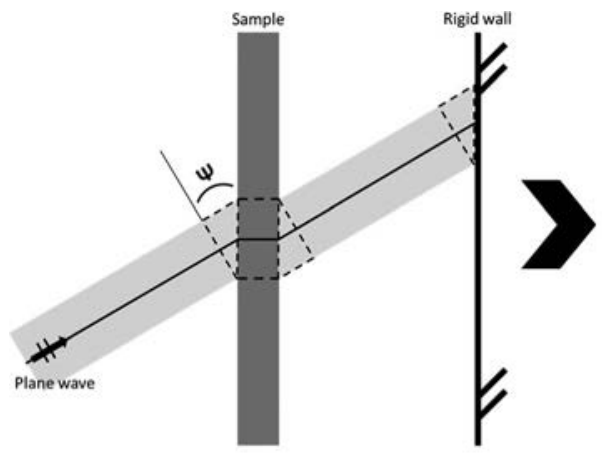

Schematic view of sample under oblique incidence

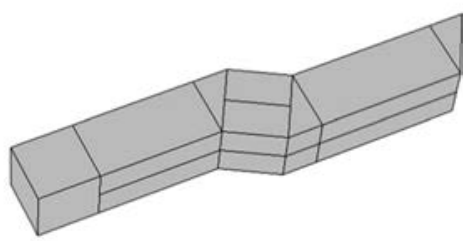

FIG. 5. Schematic view of the oblique incidence impedance tube setup for the FEM. 

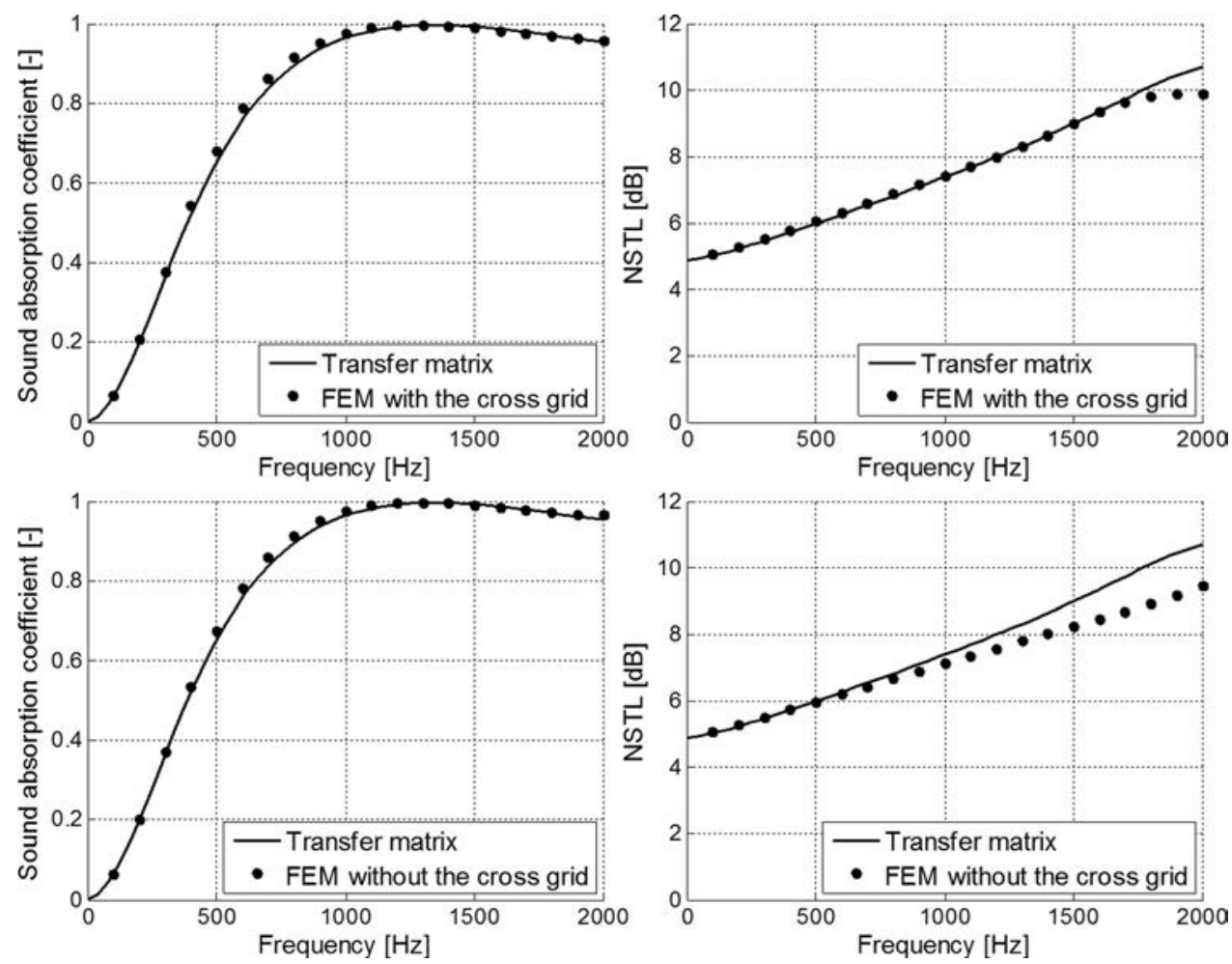

FIG. 6. Oblique $\left(30^{\circ}\right)$ incidence sound absorption coefficient and transmission loss of the parallel assembly of four materials, with the cross grid (top), and without the cross grid (bottom). Comparison between TMM and FEM results.

on the rigid wall. To evaluate the absorption coefficient and transmission loss, the three microphone method described in Sec. III is used. This oblique incidence arrangement has already been used experimentally by Mei et al. ${ }^{15}$ to estimate absorption coefficient of a metamaterial under an oblique incidence plane wave; however, the transmission loss was not evaluated.

With the aforementioned FEM model, results are obtained with and without the cross grid at an angle of incidence of $30^{\circ}$. The FEM results with and without the grid are compared to the TMM results in Fig. 6. Apart from frequencies above $1600 \mathrm{~Hz}$ (see discussion above), the TMM correlates with the cross grid FEM case. However, for the case without the grid, the discrepancy observed in the transmission loss can be explained by the fact that there is a weak diffusion between materials. For more about the diffusion phenomenon, the reader should refer to Sec. IV C. One can notice, even without the impervious walls between materials, the absorption coefficient is in line with the TMM results. This is probably due to the fact that the diffusion is low and the impedance mismatch between materials acts as a wall.

Overall, from the previous results, one can reasonably conclude that the proposed parallel TMM is valid for the parallel stacking of elements made of different porous materials
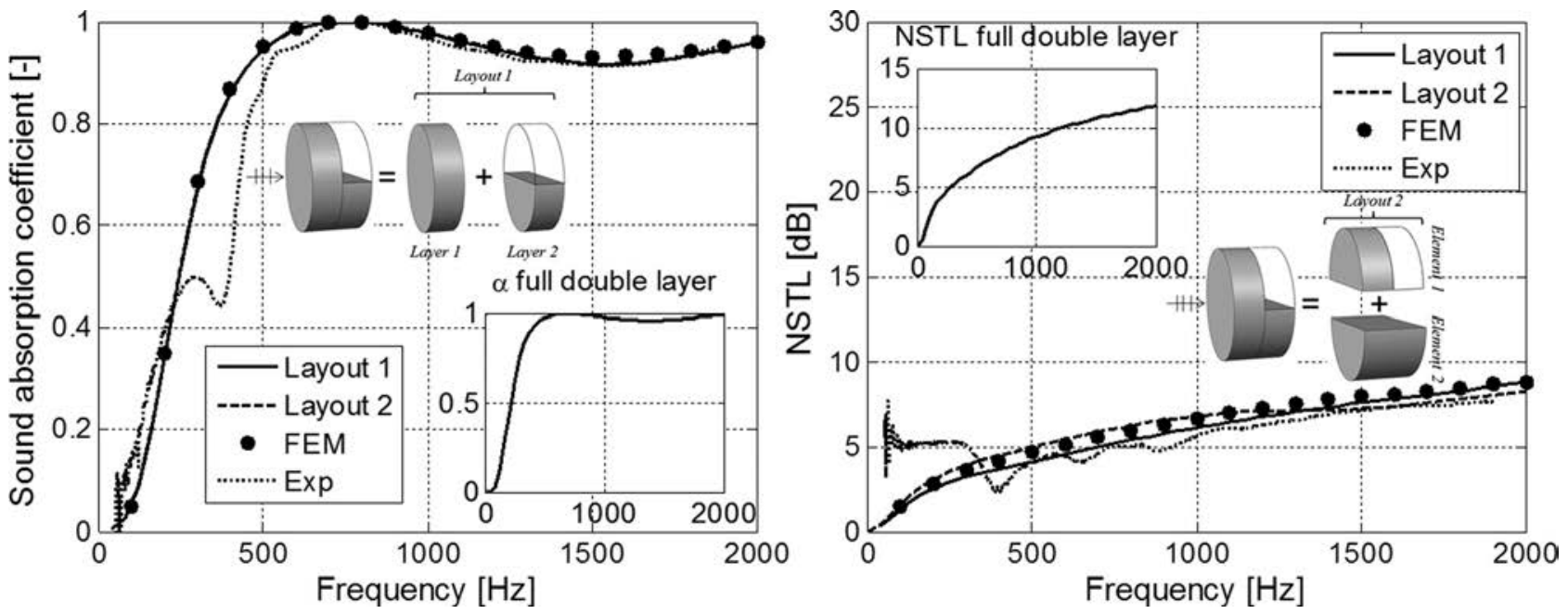

FIG. 7. Normal incidence sound absorption coefficient and transmission loss of a parallel/series patchwork of melamine foam and air. Comparison between TMM, FEM, and experimental results. Layout 1 and Layout 2 are simulated with TMM. Acoustic parameters of a full double layer are inset. 


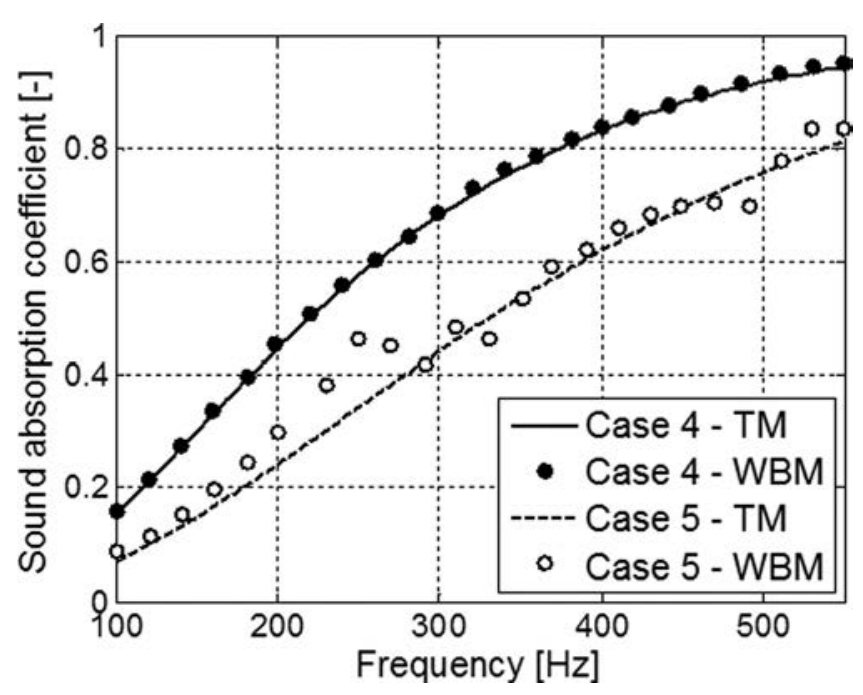

FIG. 8. Normal incidence sound absorption coefficient of the two parallel/series patchworks of melamine foam and air (case 4), and melamine foam and rigid solid (case 5). Comparison between TMM and the results of Lanoye et al. for WBM.

provided that each component is independent [assumption (3)] or diffusion between adjacent elements is weak (low permeability contrast between adjacent elements). Note that similar conclusions have been also obtained using different surface ratios.

\section{B. Parallel/series patchwork}

In this section, TMM is used to describe sound absorption coefficient and transmission loss for a two layered construction, where the first layer is a homogeneous melamine foam, and the second layer is a parallel stacking of melamine foam $(r=50 \%)$ and air $(r=50 \%)$. The construction is $100 \mathrm{~mm}$ thick $\times 100 \mathrm{~mm}$ in diameter. Figure 7 shows a schematic view of the construction, and particularly the two selected layouts for modeling. For the experimental test and FEM simulation, no rigid wall between the air and the melamine in the second layer was used. A priori, it seems contrary to assumption (3). However, it will be discussed in Sec. IV C that assumption (3) can be violated under certain circumstances.
The construction depicted in Fig. 7 can be modeled following two layouts with the TMM. The first layout consists in modeling a homogeneous layer (layer 1) in series with a parallel construction (layer 2). In that case, the global transfer matrix $\mathbf{T}_{\mathbf{G}}$ of the multilayer (referred to as layout 1) is obtained by multiplying transfer matrix $\mathbf{T}_{\mathbf{1}}$ [Eq. (2)] of layer 1 and transfer matrix $\mathbf{T}_{\mathbf{p}}$ of heterogeneous layer 2 . The second layout consists in modeling two elements in parallel. In that case, the global transfer matrix $\mathbf{T}_{\mathbf{G}}$ of the multilayer (referred to as layout 2) is obtained by the parallel assembly of transfer matrix $\mathbf{T}_{\mathbf{1}} \mathbf{T}_{\text {air }}$ of element 1 and transfer matrix $\mathbf{T}_{\mathbf{2}}$ of element 2 .

Figure 7 depicts the absorption coefficient and the transmission loss of the parallel/series configuration predicted by the TMM, the FEM, and the experiments. As one can note, the experimental results show frame resonance. Below the first frame resonance at $400 \mathrm{~Hz}$, the behavior is mainly stiffness-controlled, and above the first resonance it becomes partially mass controlled. Consequently to take into account for the mass effect of the frame above the resonance, a limp frame equivalent fluid model $^{9}$ is used for the TMM. However, if one wants to better capture the low-frequency behavior, a rigid frame model could be used below the first resonance.

Overall, the comparisons in Fig. 7 show that the TMM correlates well with both the FEM and the experiments. However, one can notice that the TMM predictions of the transmission loss of the two layouts are slightly different $( \pm 1 \mathrm{~dB})$. It is thought that this difference is associated with the fact that assumption (2) is partly violated in layout 1 in the vicinity of the interface between both layers. However, the predictions remain satisfactory with both layouts. To comfort it, in Fig. 7, the insets of acoustic parameters of a full double layer are presented. By halving the second layer, sound absorption is similar to one obtained for a full double layer with a slight decrease at $1500 \mathrm{~Hz}$ However, its impact on transmission is most important and well reproduced by the model developed TM. Halving the second layer reduces TL by approximately $3 \mathrm{~dB}$ in this case.

A similar configuration has also been studied by Lanoye et $a l{ }^{16}$ Their work is an investigation of the acoustic performance of sound absorbing patches through a numerical wave based prediction method (WBM), where its main advantage is that the sound absorbing patches do not have to

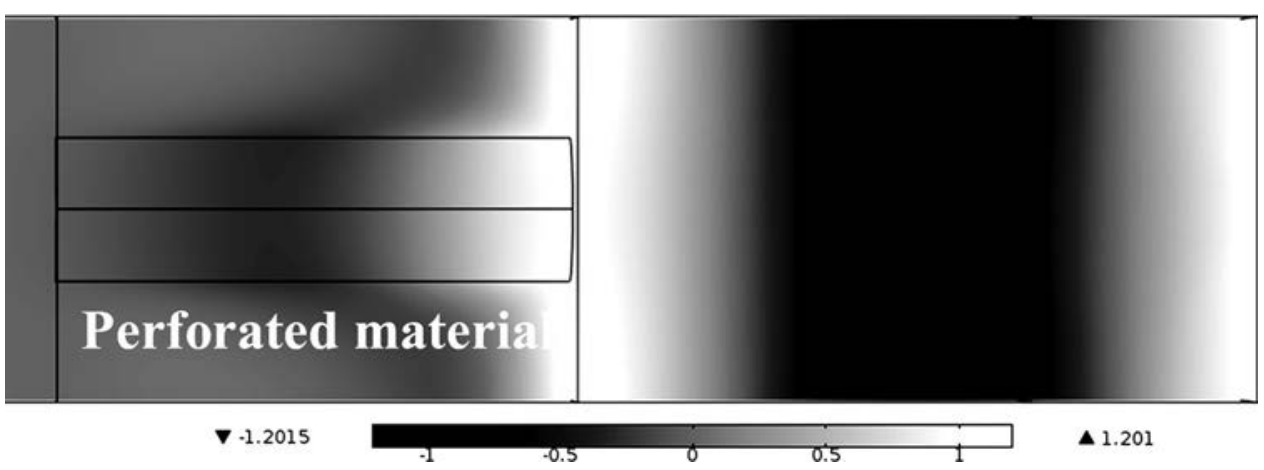

FIG. 9. Acoustic pressure field at $2000 \mathrm{~Hz}$ in the middle plane of an impedance tube (source is on the right) to highlight the diffusion phenomenon in a macro perforated rock wool sample backed by an air cavity. 

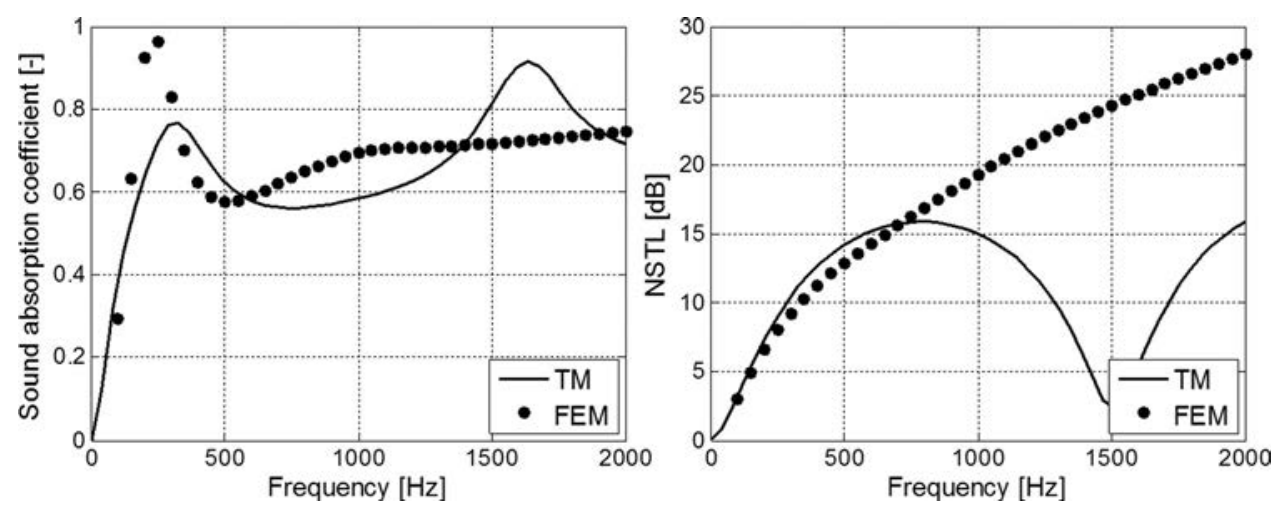

FIG. 10. Normal incidence sound absorption coefficient and transmission loss of a macro perforated rock wool of high static airflow resistivity. Comparison between TMM and FEM results.

be locally reacting. This method has been validated experimentally for a normal incidence sound absorption case in a $0.6 \times 0.6 \mathrm{~m}^{2}$ impedance tube. Figure 8 shows the comparison between the TMM and the WBM for two configurations. The first configuration is $50 \mathrm{~mm}$ thick layer of melamine foam stacked on a $50 \mathrm{~mm}$ thick parallel assembly of $50 \%$ melamine foam and 50\% air. This configuration refers to case 4 in the paper by Lanoye et al. The second configuration is similar to the first one, where the air element is replaced by a perfectly rigid element. This second configuration refers to case 5 in the paper by Lanoye et al. As can be seen in Fig. 8, the TMM correlates with the WBM; however, the TMM is more computationally efficient. Note that the melamine foam is modeled as a poroelastic material in the work by Lanoye et al.; this explains oscillations in the WBM results due to frame vibrations.

\section{Double porosity media with diffusion}

Double porosity medium can be seen as parallel constructions. Olny et al. ${ }^{13}$ have shown that for such media, interaction phenomena may occur depending upon permeability contrast. This latter is given by media size, surface ratio between media, contact shape, and distribution of the first medium with regard to the second one. Indeed, in the case of low permeability contrasts, no interaction exists.
According to Olny and Boutin, the velocity field is slightly disturbed and thus the pressure field is almost uniform. Consequently, the TMM is expected to give good results. Moreover, for low permeability contrasts, no physical rigid walls are required to isolate media, which allows new decompositions as discussed in Sec. IV B. For high permeability contrasts, the pressure and velocity fields are both disturbed and the interaction appears as diffusion phenomena. The TMM is used to attempt modeling a diffusion case. Based on a previous work published by Sgard et al., ${ }^{17}$ a rock wool block sample having a perforated hole at the center is modeled by FEM. The block is $83 \mathrm{~mm}$ high, $83 \mathrm{~mm}$ wide, and $115 \mathrm{~mm}$ thick, and the perforation diameter is $32 \mathrm{~mm}$. Figure 9 shows the acoustic pressure field at $2000 \mathrm{~Hz}$ obtained by FEM simulation in the middle plane of the simulated impedance tube. As can be seen, this pressure is not uniform along the plane and seems to be diffused in rock wool. Figure 10 depicts the absorption coefficient and the transmission loss of this sample obtained by TMM and FEM simulations. It is clear that data obtained by FEM and TMM are not in line. It is also interesting to note that the transmission loss obtained by TMM exhibits a drop at around $1500 \mathrm{~Hz}$ corresponding to the perforation length. It can be explained by the fact that the perforated rockwool behaves as an expansion chamber because TMM creates a virtual impervious wall between air and rockwool and their
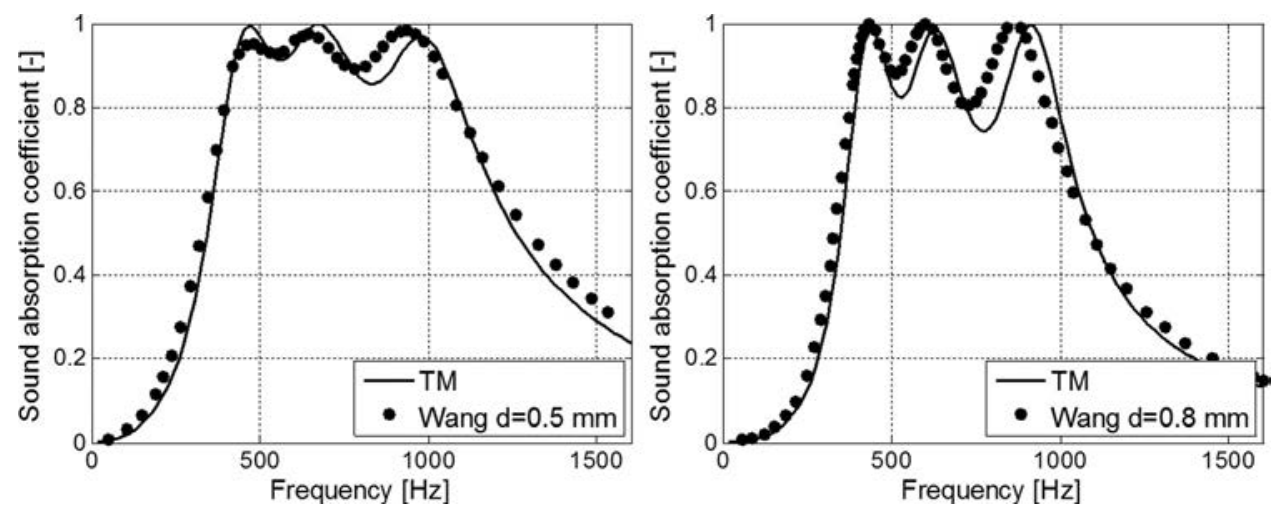

FIG. 11. Normal incidence sound absorption coefficient of the MPP absorber array consisting of a MPP of $1 \%$ perforation ratio, $0.5 \mathrm{~mm}$ thick, and orifice diameter $d$. The MPP is backed by three parallel air gap of thickness 25,50 , and $100 \mathrm{~mm}$, respectively. Comparison between TMM and Wang and Huang results for two orifice diameters: $0.5 \mathrm{~mm}$ (left) and $0.8 \mathrm{~mm}$ (right). 


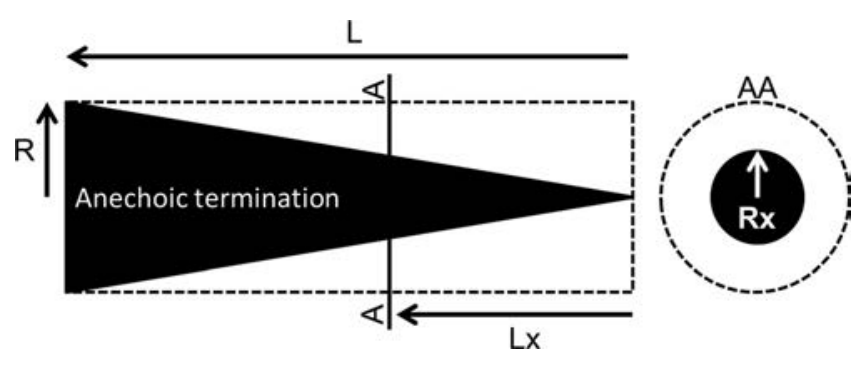

FIG. 12. Geometrical description of the anechoic cone.

contrast is very important: The perforation in the rockwool appears as a cylinder with an impervious wall which reduces the section of the impedance tube.

In conclusion, if no impervious walls isolate the parallel elements of a construction, the TMM, as defined in this paper, is valid as long as permeability contrast is low. This explains why the TMM gave good results in Secs. IV A and IV B when the isolating grid was removed. In fact, the permeability contrast between air and melamine foam elements is low.

\section{MPP absorber array}

Wang and Huang ${ }^{18}$ have investigated acoustic properties of three parallel-arranged MPP absorbers with different cavity depths. Analytical method, FEM, and experimental data have been compared with samples under normal incidence. The three cavity depths were 25, 50, and $100 \mathrm{~mm}$. Each panel occupies one-third of the total surface. The MPP is characterized by an orifice diameter $d$, a thickness of $0.5 \mathrm{~mm}$, and a perforation ratio of $1 \%$. The TMM formulation consists in a parallelization of a series of transfer matrices made of MPPs with air cavity. Figure 11 compares the TMM to the results of Wang and Huang for two MPPs, one with an orifice diameter $d=0.5 \mathrm{~mm}$, and one with $d=0.8 \mathrm{~mm}$. As expected, TMM and Wang and Huang results are in good agreement. If the setup of the model and computation time are taken into account, the proposed parallel TMM is by far quicker than FEM simulations in this case.

\section{E. Simulation of 3D configurations}

Sections IV A to IV D have been devoted to the validation and limitations of the proposed parallel TMM

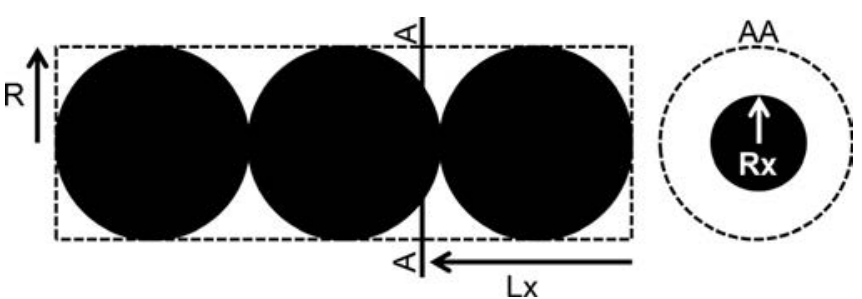

FIG. 14. Geometrical description of the three spheres of melamine foam in series.

through examples. It has been shown that series and parallel matrices can be combined in order to describe a sample made of heterogeneous layers. Thus a question arises: Is it possible to describe a $3 D$ configuration with the parallel/series TMM? To answer this question, a volume can be discretized into elementary or representative volumes as in a finite element concept. The surface of each elementary volume can be decomposed into several parallel cells. To reconstruct the entire configuration, it is necessary to stack, in series, the elementary volumes describing several materials stacked in parallel. To illustrate this approach two examples are presented below.

\section{Example 1: Anechoic cone}

The first example is an anechoic cone made of melamine foam. As previously explained, to model this 3D configuration by TMM, the volume must be discretized in many serial layers and parallel elements. For the parallel elements, the surface ratios of each constituent must be determined. Figure 12 gives all the geometrical details required for the modeling. An anechoic cone can be considered as a stack of disks, where the radius of each disk can be expressed as a function of the distance from the cone summit by trigonometric relations. At distance $L_{x}$, the surface areas of melamine and air are respectively given by

$$
\left\{\begin{array}{l}
S_{\text {mela }}\left(L_{x}\right)=\pi R_{x}^{2}=\pi \frac{R^{2}}{L^{2}} L_{x}^{2} \\
S_{\text {air }}\left(L_{x}\right)=\pi R^{2}\left(1-\frac{L_{x}^{2}}{L^{2}}\right) .
\end{array}\right.
$$
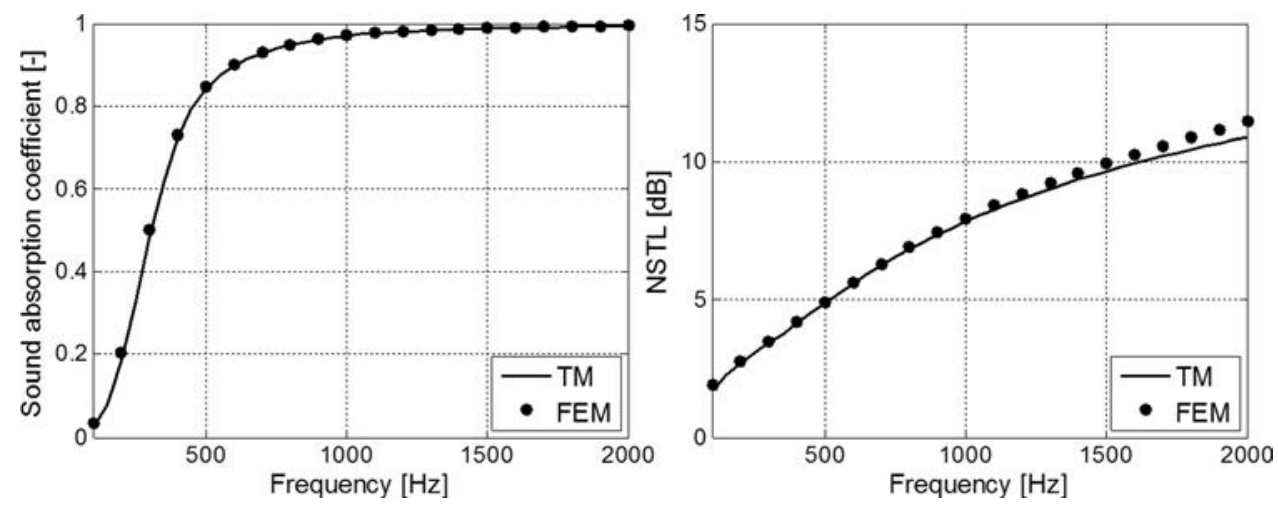

FIG. 13. Normal incidence sound absorption coefficient and transmission loss of the anechoic cone of melamine foam. The cone is $100 \mathrm{~mm}$ long and $100 \mathrm{~mm}$ in diameter. Comparison between TMM and FEM results. 

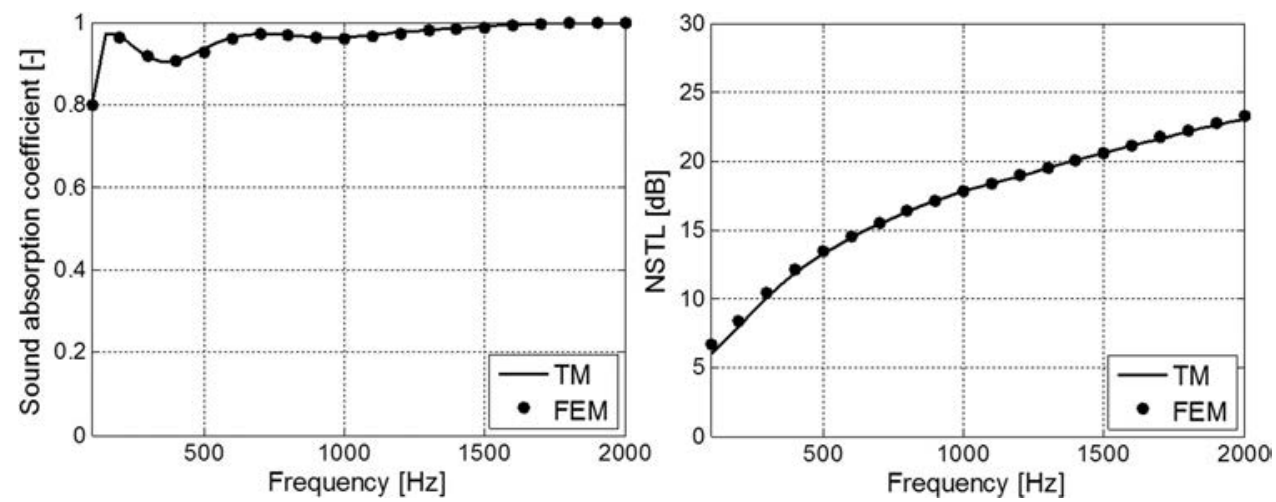

FIG. 15. Normal incidence sound absorption coefficient and transmission loss of the three spheres of melamine foam in series. Each sphere is $100 \mathrm{~mm}$ in diameter. Comparison between TMM and FEM results.

The cone is divided into 45 layers which correspond to the minimum discretization where TMM resolution converges. A 3D FEM model was used to evaluate the absorption coefficient and the transmission loss of the anechoic cone following the method described in Sec. III. Figure 13 compares the TMM and FEM results for a $300 \mathrm{~mm}$ long and $100 \mathrm{~mm}$ in diameter anechoic cone. As noted, both methods are in agreement.

\section{Example 2: Three melamine spheres in series}

The second example is a series of three spheres of melamine foam of $100 \mathrm{~mm}$ in diameter as shown in Fig. 14. As for the cone, a sphere is made of disk stacking where the radius is a function of distance from the sphere extremities. In this case, the surface ratio is periodical from one sphere to another. At distance $L_{x}$, the melamine and air surface areas are respectively given by

$$
\left\{\begin{array}{l} 
\begin{cases}S_{\text {mela }}\left(L_{x}\right)=\pi R_{x}^{2}=\pi\left(R^{2}-\left(L_{x}-R\right)^{2}\right) & \text { if } L_{x} \leq 2 R \\
S_{\text {mela }}\left(L_{x}\right)=\pi R_{x}^{2}=\pi\left(R^{2}-\left(L_{x}-2 R-R\right)^{2}\right) & \text { if } L_{x}>2 R \text { and } L_{x} \leq 4 R \\
S_{\text {mela }}\left(L_{x}\right)=\pi R_{x}^{2}=\pi\left(R^{2}-\left(L_{x}-4 R-R\right)^{2}\right) & \text { if } L_{x}>4 R \text { and } L_{x} \leq 6 R\end{cases} \\
S_{\text {air }}\left(L_{x}\right)=\pi R^{2}-S_{\text {mela }}\left(L_{x}\right) .
\end{array}\right.
$$

To yield convergence of the TMM, each sphere was divided into 35 layers. A 3D FEM model was used to evaluate the absorption coefficient and the transmission loss of the spheres of melamine foam in series. Figure 15 presents the results obtained with TMM and FEM. Once again, both methods give similar results.

The two previous 3D configurations showed that the proposed parallel TMM coupled to the classical serial TMM can handle 3D problems not possible to handle with the classical TMM only. In fact, the classical TMM is limited to laterally infinite and homogeneous multilayer samples. Consequently, the proposed approach could be referred to as an element transfer matrix method (ETMM). It is important to highlight that the computation time of this ETMM is a fraction of that of the FEM.

\section{CONCLUSION}

An extension to the TMM has been developed to handle a parallel assembly of multiple elements. It has been shown that the parallel TMM compares well with experimental and FEM results on 2D or 3D configurations. The method relies on five main assumptions and it has been shown that some assumptions may not be satisfied. However, this does not necessarily affect the accuracy of the predictions made with the proposed method. One of the key assumptions is that there is no pressure diffusion between adjacent parallel elements. It has been shown than when the permeability contrast between adjacent elements is weak, the proposed parallel TMM works. However, for high permeability contrasts, the method clearly fails. Also, the approach has been able to handle more complex 3D configurations of finite lateral dimensions following a 3D discretization of the geometry. In this way, similar to the FEM, the proposed method becomes an ETMM. Such an ETMM can be useful to optimize and analyze heterogeneous structured surfaces and volumes which are characteristic to acoustic metamaterials.

\section{ACKNOWLEDGMENT}

This work was supported by grants-in-aid from the Natural Sciences and Engineering Research Council of Canada (N.S.E.R.C.).

${ }^{1}$ J. F. Allard and N. Atalla, Propagation of Sound in Porous Media: Modeling Sound Absorbing Materials, 2nd ed. (Elsevier Applied Science, New York, 2009), pp. 243-307. 
${ }^{2}$ M. Yairi, K. Sakagami, K. Takebayashi, and M. Morimoto, "Excess sound absorption at normal incidence by two microperforated panel absorbers with different impedance," Acoust. Sci. Technol. 32, 194-200 (2011).

${ }^{3}$ N. K. Vijayasree and M. L. Munjal, "On an Integrated Transfer Matrix method for multiply connected mufflers," J. Sound Vib. 331, 1926-1938 (2012).

${ }^{4} \mathrm{~J}$. Wang, P. Leistner, and X. Li, "Prediction of sound absorption of a periodic groove structure with rectangular profile," Appl. Acoust. 73, 960-968 (2012).

${ }^{5}$ J. Ducourneau, L. Bos, V. Planeau, A. Faiz, S. S. Lami, and A. Nejade, "Prediction of the acoustic pressure above periodically uneven facings in industrial workplaces,” J. Sound Vib. 329, 2276-2290 (2010).

${ }^{6}$ N. Atalla, R. Panneton, and J. F. Allard, "Sound absorption by non homogeneous thin porous layers,” Acta. Acust. Acust. 83, 891-896 (1997).

${ }^{7}$ S. Ghosh, Network Theory: Analysis and Synthesis (Prentice-Hall of India Learning, New Delhi, 2005), pp. 346-403.

${ }^{8}$ T. Dupont, P. Leclaire, O. Sicot, X. L. Gong, and R. Panneton, "Acoustic properties of air saturated porous materials containing dead-end porosity," J. Appl. Phys. 110, 094903 (2011).

${ }^{9}$ R. Panneton, "Comments on the limp frame equivalent fluid model for porous media," J. Acoust. Soc. Am. 122, EL217-222 (2007).

${ }^{10}$ Y. Salissou, R. Panneton, and O. Doutres, "Complement to standard method for measuring normal incidence sound transmission loss with three microphones,” J. Acoust. Soc. Am. 131, EL216-EL222 (2012).
${ }^{11}$ J. F. Allard, B. Brouard, D. Lafarge, and W. Lauriks, "Reciprocity and antireciprocity in sound transmission through layered materials including elastic and porous media," Wave Motion 17, 329-335 (1993).

${ }^{12}$ O. Doutres, Y. Salissou, N. Atalla, and R. Panneton, "Evaluation of the acoustic and nonacoustic properties of sound absorbing materials using a three-microphone impedance tube," Appl. Acoust. 71, 506-509 (2010).

${ }^{13} \mathrm{X}$. Olny and C. Boutin, "Acoustic wave propagation in double porosity media," J. Acoust. Soc. Am. 114, 73-89 (2003).

${ }^{14}$ ASTM E2611-09: Standard Test Method for Measurement of Normal Incidence Sound Transmission of Acoustical Materials Based on the Transfer Matrix Method (American Society for Testing and Materials, New York, 2009).

${ }^{15}$ J. Mei, G. Ma, M. Yang, Z. Yang, W. Wen, and P. Sheng, "Dark acoustic metamaterials as super absorbers for low-frequency sound," Nat. Commun. 3, 576 (2012)

${ }^{16}$ R. Lanoye, G. Vermeir, W. Lauriks, F. Sgard, and W. Desmet, "Prediction of the sound field above a patchwork of absorbing materials," J. Acoust. Soc. Am. 123, 793-802 (2008).

${ }^{17}$ F. C. Sgard, X. Olny, N. Atalla, and F. Castel, "On the use of perforations to improve the sound absorption of porous materials," Appl. Acoust. 66, 625-651 (2005)

${ }^{18} \mathrm{C}$. Wang and L. Huang, "On the acoustic properties of parallel arrangement of multiple micro perforated panel absorbers with different cavity depths," J. Acoust. Soc. Am. 130, 208-218 (2011). 